\title{
TIME-DEPENDENT VECTOR STABILIZATION
}

\author{
SERGEY NIKITIN
}

Received 1 February 2005; Revised 13 April 2006; Accepted 25 June 2006

The paper presents necessary and sufficient conditions for a single input system defined by a time-dependent vector to be stabilized by a linear feedback. The paper employs a new technique based on averaging along a moving time interval.

Copyright (c) 2006 Hindawi Publishing Corporation. All rights reserved.

\section{Introduction}

Stabilization is one of the central themes of control theory. It was shown in [1-4] that the class of continuous stationary feedbacks is too restrictive for the purposes of stabilization of nonlinear systems. In other words, in order to design a continuous feedback stabilizer one needs to use functions depending on time and state $[6,8]$. To stay with the class of stationary feedbacks one has to deal with piecewise continuous functions [5]. The synthesis procedures for both continuous and piecewise continuous stabilizers are developed only for some special types of systems. For example, in $[5,4]$ it is shown how to construct stationary piecewise continuous stabilizers for generic two-dimensional affine nonlinear systems. On the other hand, the papers $[6,8,9]$ show how to design feedbacks for certain types of nonholonomic systems. Both approaches (nonstationary continuous and stationary discontinuous) are quite complicated as far as the feedback synthesis is concerned. The statement of the problem considered in this paper is not new. There are many publications related to the problems that are similar in nature to the stabilization of a single time-dependent vector. Similar equations arise naturally in learning systems, repetitive, and adaptive control systems. That underlines the importance of the approach presented here. Not only the main result but especially the technique employed in this publication might have consequences of significant magnitude for various branches of applied mathematics. Novelty of the approach developed in this paper is due to averaging the time-dependent vector field over a time interval. By its nature this approach does not impose any unnatural restrictions on the vector field in question. That strongly advocates its advantage and power. The time-averaging technique is not new itself. In fact equity traders use it daily comparing dynamics of 200- and 50-day moving averages for

Hindawi Publishing Corporation

International Journal of Mathematics and Mathematical Sciences

Volume 2006, Article ID 34569, Pages 1-6

DOI 10.1155/IJMMS/2006/34569 
prices of securities. However, the mathematical analysis of this idea and its illustration by the solution of a classical control problem is completely new. Though the method presented by this paper is discussed only in the framework of a single time-dependent vector, its core ideas are of general nature and will be of great value for system theory. Moreover, the new technical approach developed in this paper is of fundamental importance and its further study might promise new advances not only in development of control systems but also in analysis of general dynamics.

This paper presents a new effective feedback synthesis for stabilization of a timedependent single vector. The proposed synthesis procedure gives us time-dependent linear stabilizers. The paper formulates necessary and sufficient conditions for such stabilizer to make the corresponding closed loop system asymptotically stable.

\section{Preliminaries}

Consider a system

$$
\dot{x}=b(t) \cdot u
$$

where $u$ is the control input; $x$ denotes the state of the system and $x \in \mathbb{R}^{n}, \mathbb{R}^{n}$ denotes $n$-dimensional linear real space. $b(t)$ is a continuous time-dependent vector field such that

$$
\forall t \in \mathbb{R} \quad b(t) \in \mathbb{R}^{n}
$$

Throughout the paper we assume that $\mathbb{R}^{n}$ is equipped with the scalar product and $\|x\|$ denotes the magnitude of $x$, that is, $\|x\|=\sqrt{\langle x, x\rangle}$, where $\langle x, x\rangle$ is the scalar product of $x$ with itself.

The goal of this paper is to find necessary and sufficient conditions for the linear feedback

$$
u=-\langle b(t), x\rangle
$$

to stabilize the system (2.1). The stabilization is defined as follows.

Definition 2.1. The system (2.1) is said to be stabilizable by the feedback (2.3) if the solution $x\left(t, x_{0}\right)$ of the closed loop system

$$
\dot{x}=-b(t) \cdot\langle b(t), x\rangle, \quad x(0)=x_{0},
$$

tends to zero as $t$ goes to infinity,

$$
\lim _{t \rightarrow \infty} x\left(t, x_{0}\right)=0 \quad \forall x_{0} \in \mathbb{R}^{n},
$$

and the equilibrium $x=0$ is stable.

\section{Necessary and sufficient conditions}

Our main goal is to find conditions that guarantee that the linear feedback (2.3) stabilizes the system (2.1). In other words, we need to study asymptotic stability (in large) of the 
origin for the closed loop system

$$
\dot{x}=-b(t) \cdot b^{T}(t) x
$$

where $b^{T}(t), b(t)$ denote the row and column, respectively. If the feedback (2.3) stabilizes the system (2.1), then

$$
\lim _{t \rightarrow \infty}\left\|x\left(t, x_{0}\right)\right\|^{2}=0 \quad \forall x_{0} \in \mathbb{R}^{n}
$$

Hence, for any fixed real positive number $\epsilon$ we have

$$
\lim _{t \rightarrow \infty} \int_{0}^{\epsilon} \frac{\epsilon-\tau}{2}\left\|x\left(t+\tau, x_{0}\right)\right\|^{2} d \tau=0 \quad \forall x_{0} \in \mathbb{R}^{n} .
$$

Consider more closely the integral

$$
\int_{0}^{\epsilon} \frac{\epsilon-\tau}{2}\left\|x\left(t+\tau, x_{0}\right)\right\|^{2} d \tau
$$

It follows from

$$
\frac{d}{d t}\left\|x\left(t, x_{0}\right)\right\|^{2}=-2 \cdot\left\langle b(t), x\left(t, x_{0}\right)\right\rangle^{2} \leq 0
$$

that $\left\|x\left(t_{1}, x_{0}\right)\right\|^{2} \geq\left\|x\left(t_{2}, x_{0}\right)\right\|^{2}$ for $t_{2}>t_{1}$. That means

$$
\int_{0}^{\epsilon} \frac{\epsilon-\tau}{2}\left\|x\left(t+\tau, x_{0}\right)\right\|^{2} d \tau \geq\left\|x\left(t+\epsilon, x_{0}\right)\right\|^{2} \cdot \int_{0}^{\epsilon} \frac{\epsilon-\tau}{2} d \tau=\left\|x\left(t+\epsilon, x_{0}\right)\right\|^{2} \cdot \frac{\epsilon^{2}}{4} .
$$

If we find conditions that guarantee

$$
\int_{0}^{\epsilon} \frac{\epsilon-\tau}{2}\left\|x\left(t+\tau, x_{0}\right)\right\|^{2} d \tau \longrightarrow 0 \quad \text { as } t \longrightarrow \infty,
$$

then that will imply that the linear feedback (2.3) stabilizes the system (2.1).

Differentiating the integral

$$
\int_{0}^{\epsilon} \frac{\epsilon-\tau}{2}\left\|x\left(t+\tau, x_{0}\right)\right\|^{2} d \tau
$$

with respect to time yields

$$
\frac{d}{d t} \int_{0}^{\epsilon} \frac{\epsilon-\tau}{2}\left\|x\left(t+\tau, x_{0}\right)\right\|^{2} d \tau=-\int_{0}^{\epsilon}(\epsilon-\tau)\left\langle x\left(t+\tau, x_{0}\right), b(t+\tau) \cdot b^{T}(t+\tau) x\left(t+\tau, x_{0}\right)\right\rangle d \tau .
$$

Integrating by parts leads us to the following important formula:

$$
\frac{d}{d t} \int_{0}^{\epsilon} \frac{\epsilon-\tau}{2}\left\|x\left(t+\tau, x_{0}\right)\right\|^{2} d \tau=-\int_{0}^{\epsilon}\left\langle x\left(t+\tau, x_{0}\right), A(t, \tau) x\left(t+\tau, x_{0}\right)\right\rangle d \tau,
$$


4 Time-dependent vector stabilization

where

$$
\begin{aligned}
A(t, \tau)= & \int_{0}^{\tau} b(t+\theta) \cdot b^{T}(t+\theta)+(\epsilon-\tau) \\
& \cdot\langle b(t+\theta), b(t+\tau)\rangle \cdot\left(b(t+\theta) \cdot b^{T}(t+\tau)+b(t+\tau) \cdot b^{T}(t+\theta)\right) d \theta .
\end{aligned}
$$

Let

$$
\lambda_{\min }(t, \tau), \quad \lambda_{\max }(t, \tau)
$$

denote minimal and maximal eigenvalues of $A(t, \tau)$. Then the next theorem gives us necessary and sufficient conditions for the system (2.1) to be stabilized by the linear feedback (2.3). Notice that one is assured that $\lambda_{\min }(t, \tau)$ and $\lambda_{\max }(t, \tau)$ are continuous functions of the variables $t$ and $\tau$ by well-known general results on the dependence of the eigenvalues of a parametric symmetric matrix on the entries of that matrix. It then follows that the integral expressions in the next theorem are well defined.

THeOREM 3.1. If there exists a real number $\epsilon>0$ such that

$$
\limsup _{t \rightarrow \infty} \int_{0}^{t} \int_{0}^{\epsilon} \lambda_{\min }(\nu, \tau) d \tau d \nu=\infty,
$$

then the linear feedback (2.3) stabilizes the system (2.1). On the other hand, if

$$
\exists \epsilon>0, \quad \liminf _{t \rightarrow \infty} \int_{0}^{t} \int_{0}^{\epsilon} \lambda_{\max }(\nu, \tau) d \tau d \nu<\infty,
$$

then the linear feedback (2.3) does not stabilize the system (2.1).

Proof. It follows from (3.10) that

$$
\begin{aligned}
\int_{0}^{\epsilon} \frac{\epsilon-\tau}{2}\left\|x\left(t+\tau, x_{0}\right)\right\|^{2} d \tau \leq & -\int_{0}^{t} \int_{0}^{\epsilon} \lambda_{\min }(\nu, \tau)\left\|x\left(\nu+\tau, x_{0}\right)\right\|^{2} d \tau d \nu \\
& +\int_{0}^{\epsilon} \frac{\epsilon-\tau}{2}\left\|x\left(\tau, x_{0}\right)\right\|^{2} d \tau
\end{aligned}
$$

Due to monotonicity of $\left\|x\left(t, x_{0}\right)\right\|^{2}$ we have

$$
\frac{\epsilon^{2}}{4}\left\|x\left(t+\epsilon, x_{0}\right)\right\|^{2} \leq-\int_{0}^{t}\left(\int_{0}^{\epsilon} \lambda_{\min }(\nu, \tau) d \tau\right)\left\|x\left(\nu+\epsilon, x_{0}\right)\right\|^{2} d \nu+\frac{\epsilon^{2}}{4}\left\|x_{0}\right\|^{2} .
$$

It follows from the very well-known Gronwall inequality (see, e.g., [7]) that

$$
\left\|x\left(t+\epsilon, x_{0}\right)\right\|^{2} \leq\left\|x_{0}\right\|^{2} \cdot \exp \left\{-\frac{4}{\epsilon^{2}} \int_{0}^{t} \int_{0}^{\epsilon} \lambda_{\min }(\nu, \tau) d \tau d \nu\right\} .
$$

Thus,

$$
\limsup _{t \rightarrow \infty} \int_{0}^{t} \int_{0}^{\epsilon} \lambda_{\min }(\nu, \tau) d \tau d \nu=\infty
$$

implies that the linear feedback (2.3) stabilizes the system (2.1). 
On the other hand, (3.10) leads us to

$$
\begin{aligned}
\int_{0}^{\epsilon} \frac{\epsilon-\tau}{2}\left\|x\left(t+\tau, x_{0}\right)\right\|^{2} d \tau \geq & -\int_{0}^{t} \int_{0}^{\epsilon} \lambda_{\max }(\nu, \tau)\left\|x\left(\nu+\tau, x_{0}\right)\right\|^{2} d \tau d \nu \\
& +\int_{0}^{\epsilon} \frac{\epsilon-\tau}{2}\left\|x\left(\tau, x_{0}\right)\right\|^{2} d \tau
\end{aligned}
$$

Since the derivative $(d / d t)\left\|x\left(t, x_{0}\right)\right\|^{2}$ is not positive we have $\left\|x\left(\nu+\tau, x_{0}\right)\right\|^{2} \leq\left\|x\left(\nu, x_{0}\right)\right\|^{2}$ and

$$
\frac{\epsilon^{2}}{4}\left\|x\left(t, x_{0}\right)\right\|^{2} \geq-\int_{0}^{t}\left(\int_{0}^{\epsilon} \lambda_{\max }(\nu, \tau) d \tau\right)\left\|x\left(\nu, x_{0}\right)\right\|^{2} d \nu+\frac{\epsilon^{2}}{4}\left\|x\left(\epsilon, x_{0}\right)\right\|^{2}
$$

for $t \geq \epsilon$. After solving this inequality we obtain

$$
\left\|x\left(t, x_{0}\right)\right\|^{2} \geq\left\|x\left(\epsilon, x_{0}\right)\right\|^{2} \cdot \exp \left\{-\frac{4}{\epsilon^{2}} \int_{0}^{t} \int_{0}^{\epsilon} \lambda_{\max }(\nu, \tau) d \tau d \nu\right\}
$$

for $t \geq \epsilon$. Hence, if

$$
\exists \epsilon>0, \quad \liminf _{t \rightarrow \infty} \int_{0}^{t} \int_{0}^{\epsilon} \lambda_{\max }(\nu, \tau) d \tau d \nu<\infty,
$$

then the linear feedback (2.3) does not stabilize the system (2.1).

Notice that we owe the success in proving Theorem 3.1 to the new idea that suggests to consider

$$
\int_{0}^{\epsilon} \frac{\epsilon-\tau}{2}\left\|x\left(t+\tau, x_{0}\right)\right\|^{2} d \tau
$$

instead of $\left\|x\left(t, x_{0}\right)\right\|^{2}$. This approach seems to have further important consequences not only for control theory but also for studies of general dynamical systems.

\section{Example}

Theorem 3.1 finds many important applications in control theory. This section illustrates how one can use Theorem 3.1 to analyze and design feedback stabilizers for a twodimensional system defined by a periodic time-dependent vector field.

Consider the system

$$
\dot{x}=\left(\begin{array}{c}
1 \\
\sin (t)
\end{array}\right) u \text {. }
$$

In order to stabilize the system at the origin, one can choose the linear feedback

$$
u(t, x)=-\left(x_{1}+\sin (t) \cdot x_{2}\right)
$$


In order to prove that this feedback stabilizes system (4.1) at the origin, we will employ Theorem 3.1. The matrix $A(t, \tau)$ takes the form

$$
\begin{aligned}
& A(t, \tau)=\int_{0}^{\tau}\left(\begin{array}{c}
1 \\
\sin (t+\theta)
\end{array}\right) \cdot\left(\begin{array}{ll}
1 & \sin (t+\theta)
\end{array}\right)+(\epsilon-\tau) \cdot(1+\sin (t+\theta) \sin (t+\tau))
\end{aligned}
$$

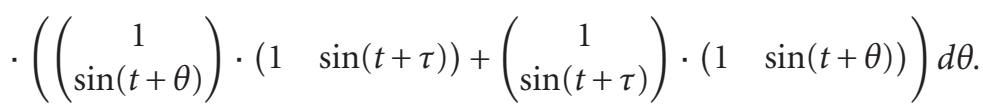

After calculating the integral $\int_{0}^{t} A(\nu, \tau) d \nu$ we obtain

$$
\int_{0}^{t} A(\nu, \tau) d \nu=t \cdot\left(\begin{array}{cc}
\tau+\alpha(\tau, \epsilon) & 0 \\
0 & \frac{\tau}{2}+\beta(\tau, \epsilon)
\end{array}\right)+O\left(\frac{1}{t}\right) \quad \text { as } t \longrightarrow \infty
$$

where the functions $\alpha(\tau, \epsilon)$ and $\beta(\tau, \epsilon)$ are not negative. Hence, by Theorem 3.1 feedback (4.2) stabilizes system (4.1).

\section{Acknowledgment}

The author is very grateful to the referee who made many important suggestions that allowed to improve the formulation of the main result and the style of the paper.

\section{References}

[1] D. Aeyels, Stabilization of a class of nonlinear systems by a smooth feedback control, Systems \& Control Letters 5 (1985), no. 5, 289-294.

[2] J.-M. Coron, A necessary condition for feedback stabilization, Systems \& Control Letters 14 (1990), no. 3, 227-232.

[3] S. Nikitin, Topological necessary conditions of smooth stabilization in the large, Systems \& Control Letters 21 (1993), no. 1, 35-41.

[4] _ Global Controllability and Stabilization of Nonlinear Systems, Series on Advances in Mathematics for Applied Sciences, vol. 20, World Scientific, New Jersey, 1994.

[5] , Piecewise-constant stabilization, SIAM Journal on Control and Optimization 37 (1999), no. 3, 911-933.

[6] J.-B. Pomet, Explicit design of time-varying stabilizing control laws for a class of controllable systems without drift, Systems \& Control Letters 18 (1992), no. 2, 147-158.

[7] N. Rouche, P. Habets, and M. Laloy, Stability Theory by Liapunov's Direct Method, Applied Mathematical Sciences, vol. 22, Springer, New York, 1977.

[8] C. Samson, Control of chained systems application to path following and time-varying pointstabilization of mobile robots, IEEE Transactions on Automatic Control 40 (1995), no. 1, 64-77.

[9] O. J. Sørdalen and O. Egeland, Exponential stabilization of nonholonomic chained systems, IEEE Transactions on Automatic Control 40 (1995), no. 1, 35-49.

Sergey Nikitin: Department of Mathematics and Statistics, Arizona State University, Tempe, AZ 85287-1804, USA

E-mail address: nikitin@asu.edu 


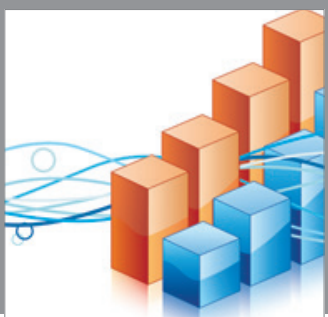

Advances in

Operations Research

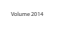

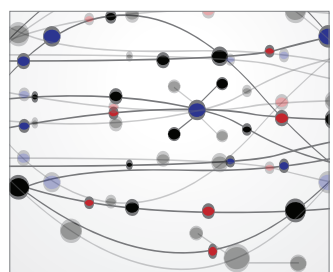

\section{The Scientific} World Journal
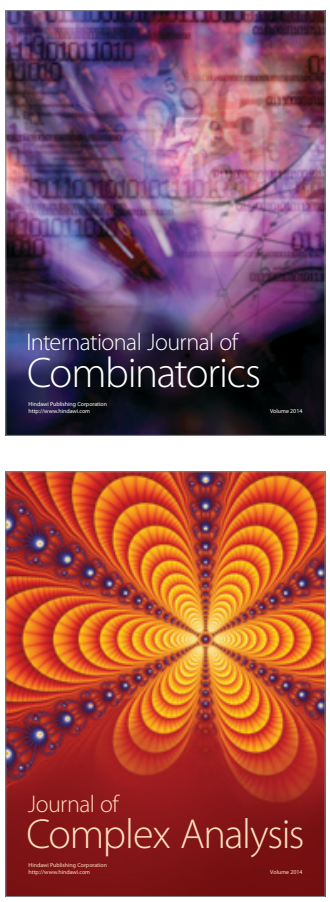

International Journal of

Mathematics and

Mathematical

Sciences
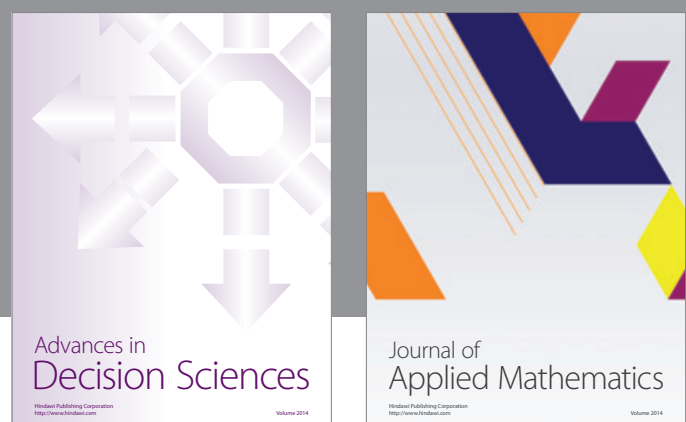

Journal of

Applied Mathematics
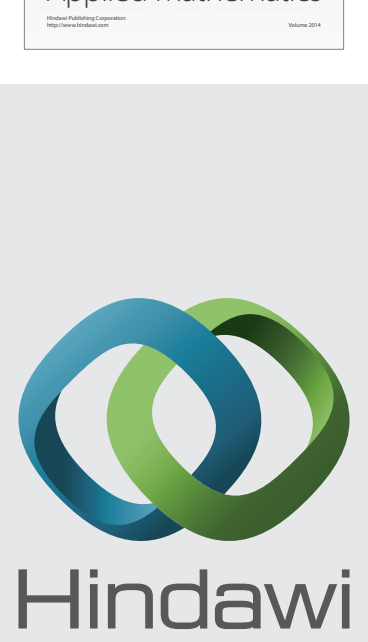

Submit your manuscripts at http://www.hindawi.com
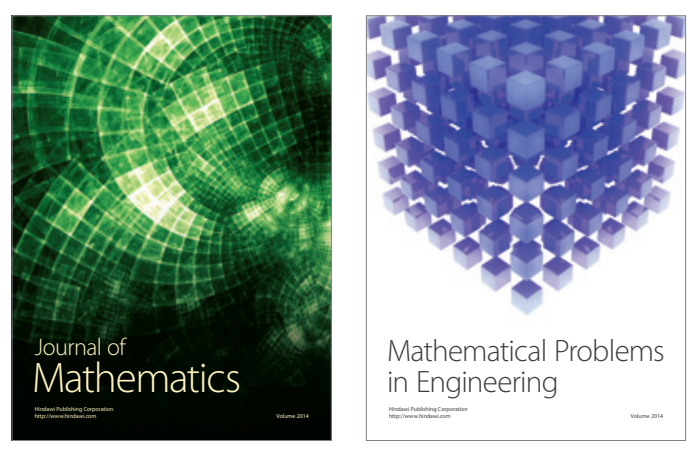

Mathematical Problems in Engineering
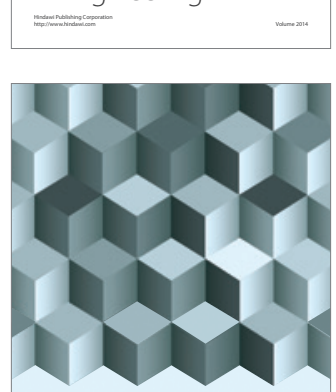

Journal of

Function Spaces
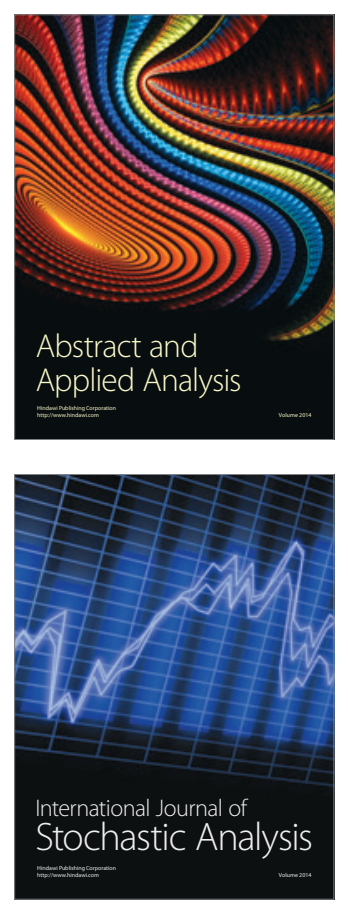

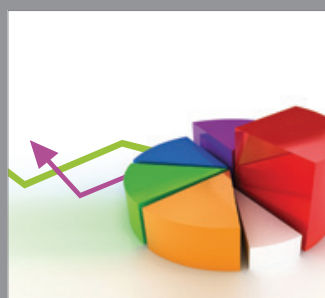

ournal of

Probability and Statistics

Promensencen
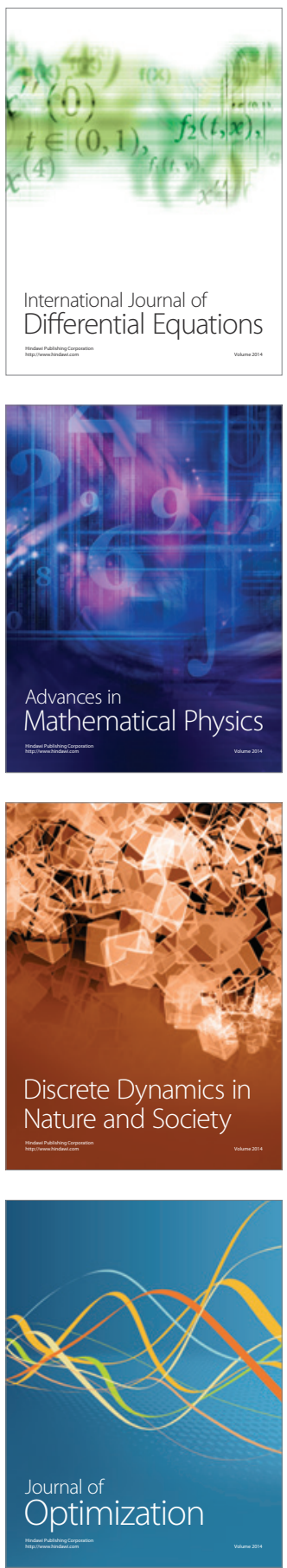